\title{
NONLINEAR NETWORKS. IV
}

\section{R. J. DUFFIN}

It may be well to point out explicitly that the networks considered in this paper and in the preceding papers are definitely not arbitrary. nonlinear networks. Rather, it has been the aim to define nonlinear networks which share the uniqueness properties of passive linear networks. Physical considerations suggest that such networks may be aptly termed reliable networks.

The networks considered here consist of transformers with ferromagnetic cores (assumed to have negligible hysteresis) and Ohmic resistors, arbitrarily interconnected to a set of generators. It is found that as in the case of linear networks, under given electromotive forces from the generators, the currents flowing are uniquely determined after sufficient time has elapsed. Moreover, if the electromotive force is periodic, a unique, periodic current flow can exist.

The proof begins by integrating the network equations with respect to time. It turns out that the integrated equations have the same form as the network equations of a network containing linear capacitors and nonlinear resistors. The differential permeability of a ferromagnetic lies between positive limits; in the integrated equations this is imaged by the condition that the differential resistance lies between positive limits. The results stated are then easily deduced from theorems developed in preceding papers for networks containing such quasi-linear resistors $[1,2] .^{1}$ (These papers will hereinafter be referred to as I and III.)

In most applications of transformers the nonlinearity of the magnetization curve is an undesirable feature; however, the operation of flux gate magnetometers, magnetic voltage regulators, frequency multipliers, and magnetic amplifiers depends essentially on the nonlinearity.

Considerable space is devoted to the concepts of a transformer and of a transformer network. In $\$ 1$ the transformers are restricted to be of a symmetrical type so that their properties can be determined fairly rigorously from elementary considerations. The general transformer is treated in $\$ 4$ by means of the hypothesis of the magnetic network. The concept of topologically coupled networks employed there will be found quite interesting from an abstract point of view.

Presented to the Society, December 30,1948; received by the editors November 16, 1948.

1 Numbers in brackets refer to the references cited at the end of the paper. 
1. Transformer networks. By a "transformer" is meant, in this section, a set of coils of insulated wire wound in uniform layers on a ring-shaped core of ferromagnetic material. A transformer network is a collection of transformers and generators, the various coils and generators being arbitrarily interconnected. Any conservative distribution of electric current in the network may be uniquely expressed as a superposition of cyclic currents $i_{1}, i_{2}, \cdots, i_{n}$ flowing in $n$ closed circuits, provided these circuits are selected so as to be a maximal linearly independent set. A positive direction of circulation is arbitrarily assigned to each circuit. Let $e_{r}$ be the sum of the electromotive forces of the generators acting in the $r$ th circuit. By Faraday's law of induction, $e_{r}=d v_{r} / d t$ where $v_{r}$ is the net magnetic flux linked with the $r$ th circuit. This assumes, of course, that these circuits have no resistance. To express equations which relate electromotive forces and currents, it is now necessary to express the magnetic fluxes as a function of the currents.

The core of each transformer will be regarded as composed of a number of co-axial sub-rings with "small" cross-sectional diameter. By the assumed geometric symmetry it follows that the tangential magnetic field $H$ is the same at every point of the sub-ring. The sign of $H$ is determined by arbitrarily prescribing a direction of circulation in the sub-ring. According to Ampere's law the line integral of the tangential component of the magnetic field around a closed path is given by the net current linked with the path. (This assumes the socalled rational units.) If the circumference of the ring is $s$, then $s H=\sum C_{k} i_{k}$. Here $C_{k}$ is the net number of times the electric circuit $k$ links the sub-ring, the sense of linkage being determined by the right-hand rule.

In ferromagnetics the flux density, $B$, satisfies the relation $B=\mu(H)$, and if $d B=\mu^{\prime} d H$, then it is an experimental fact that the differential permeability, $\mu^{\prime}$, satisfies the relation $\mu_{v} \leqq \mu^{\prime} \leqq A$. Here $\mu_{v}$ is the permeability of the vacuum. The positive constant $A$ may be taken so large that this relation is uniform for all sub-rings, and in addition, that $A^{-1} \leqq \mu_{v}$. Let $f$ be the magnetic flux flowing in the sub-ring under consideration. Then $f=a \mu\left(\sum C_{k} i_{k} s^{-1}\right)$. Here $a$ is the crosssectional area of the ring. For convenience in notation this last relation may be written $f=U\left(\sum C_{k} i_{k}\right)$ where the function $U$ has a derivative $u$ lying between positive limits.

Let the sub-rings from all the transformers be placed in an ordered sequence. The variables of the sub-rings will be distinguished by superscripts. Thus the net flux linked with the $r$ th electrical circuit is $v_{r}=\sum C_{r}^{\prime} f^{i}$. Introducing the relation for $f^{i}$ found above gives the 
desired relation

$$
v_{r}=\sum C_{r}^{j} U^{j}\left(\sum ! C_{k}^{i} i_{k}\right)
$$

Differentiating with respect to time yields

$$
e_{r}=\sum \sum u^{j} C_{r}^{j} C_{k}^{j} i_{k}^{\prime}=\sum L_{r k}^{\prime} i_{k}^{\prime}
$$

Here $i_{k}^{\prime}=d i_{k} / d t$, and the matrix $L_{r k}^{\prime}$ is defined by first summing over $j$; it may be called the inductance matrix. It is clear that $L_{r \mathrm{k}}^{\prime}$ is symmetric; moreover, it is semi-definite, as may be seen from the following relation:

$$
\sum \sum L_{r k}^{\prime} i_{r}^{\prime} i_{k}^{\prime}=\sum u^{i}\left(\sum C_{r}^{i} i_{r}^{\prime}\right)^{2} \geqq 0 .
$$

Relation (2) would of course remain valid if the core material were replaced by a material having a constant permeability of unity. Let the inductance matrix in this case be designated as $L_{r k}$; then it is clear from (3) that the following inequality holds:

$$
A^{-1} \sum \sum L_{r k} i_{r}^{\prime} i_{k}^{\prime} \leqq \sum \sum L_{r k}^{\prime} i_{r}^{\prime} i_{k}^{\prime} \leqq A \sum \sum L_{r k} i_{r}^{\prime} i_{k}^{\prime} .
$$

The following observation is of interest in the theory of linear networks. Relation (3) shows that the inductance matrix $L$ is semidefinite; this, of course, has long been known. The same formula shows that, conversely, any semi-definite matrix is the inductance matrix of some network. To see this, note that $L$ is here expreśsed in the form $L=K^{*} K$ where $K^{*}$ is the transpose of the matrix $K$. On the other hand, it is a relatively easy problem of matrix theory to show that any semi-definite matrix permits of such a factoring. The observation that an arbitrary matrix $K$ can be achieved; in principle, by a suitable coil design completes the proof. (R. M. Foster has informed the writer that he also discovered this result and presented it some time ago in his classroom lectures. Foster's proof is quite simple.)

2. Quasi-linear replacements. The nonlinear relation (1) just obtained between the flux vector, $v$, and the current vector, $i$, is of the type which was called, in I and III, a quasi-linear replacement. ${ }^{2}$

2 The following corrective paragraph should be added to the paper Nonlinear networks. III at the bottom of page 122:

"The one-dimensional mean value relation $V(x)-V(y)=V^{\prime} x-V^{\prime} y$ remains valid in $n$ dimensions if $V^{\prime}$ is interpreted as $V_{m}^{\prime}=\int_{0}^{\prime} V^{\prime}(i) d \theta$. Here $\theta$ is a scalar and $i=y$ $+\theta(x-y)$. Clearly $V_{m}^{\prime}$ is a symmetric matrix. Moreover, integrating (iii) with respect to $\theta$ shows that $V_{m}^{\prime}$ satisfies (iii). Thus $V_{m}^{\prime}$ satisfies the conditions (ii) and (iii) on $V^{\prime}$, so in what follows the subscript $m$ is deleted. While this notation causes some ambiguity, it is precisely the notation employed in Nonlinear networks. I." 
Consider, then, an abstract transformation, $v=V(i)$, of an $n$-dimensional vector space with corresponding differential transformation, $d v=V^{\prime} d i$, where $V^{\prime}$ is the matrix of differential coefficients. If $L$ is a symmetric semi-definite matrix, the transformation $V(i)$ is a quasilinear replacement of the linear transformation $L i$ if:

(i) $V(0)=0$;

(ii) $V^{\prime}(i)$ exists for all $i$ as a symmetric matrix;

(iii) there is a positive constant, $A$, such that for all vectors $i$ and $z, A^{-1}(L z, z) \leqq\left(V^{\prime} z, z\right) \leqq A(L z, z)$.

In the last line the standard notation for the inner product has been employed; that is, if $x$ and $y$ are two vectors, then $(x, y)=\sum x_{k} y_{k}$. The following simple consequence will be needed later:

Lemma. If $V(i)$ is a quasi-linear replacement of $L i$, then so also is $V_{1}(i)=V(i+k)-V(k)$ where $k$ is a constant vector.

To indicate the nature of a quasi-linear transformation, several of its properties will now be mentioned: If $M$ is the manifold determined by vectors of the form $L i$, and if $k$ is any vector perpendicular to this manifold, then $V(i+k)=V(i)$. All vectors of the form $V(i)$ are contained in $M$. If $v$ is in $M$, the relation $v=V(i)$ has a unique solution for $i$ in $M, i=W(v)$. Moreover, the domain of $W$ may be extended so that it also is a quasi-linear replacement of $L i$. Proofs of these statements are not difficult and are contained essentially in I and III.

3. Transformer resistor networks. To obtain the equations defining the flow of current in a general network containing linear transformers, linear resistors, and linear capacitors, it is simply necessary to add voltage drops due to the resistors and capacitors to the equation already obtained for the inductive voltage drops. It is convenient to introduce the vector $y$ of electric charge, which is such that $i=y^{\prime}$. Then the general network equations are

$$
L y^{\prime \prime}+R y^{\prime}+S y=e .
$$

Here $R$ is the matrix of resistors, its diagonal elements, say $R_{k k}$, being the total resistance in the $k$ th circuit. The off-diagonal, $R_{k r}$, has magnitude equal to the common resistance of the circuits $k$ and $r$, and its sign depends on the relative directions assigned to the circuits $r$ and $k$. The formalism developed in $\$ 1$ may be adapted to prove these and other properties of $R$. Interpret $C_{r}^{\prime}$ as the incidence matrix between the branches $j$ and the circuits $r$ of the graph. Thus, $C_{r}^{j}=1$ if the branch $j$ occurs in the $r$ th circuit and their senses agree, and $C_{r}^{j}=-1$ if their senses disagree. If the branch $j$ does not occur in the 
$r$ th circuit, $C_{r}^{j}=0$. In relation (1) interpret $v_{r}$ as the net voltage drop across the resistors of the $r$ th circuit, and interpret $U^{i}$ as the resistance of the $j$ th branch. Then it may be seen from relation (3) that $R$ is semi-definite. It also follows from (3) that if none of the branches have zero resistance, $R$ is definite. For quasi-linear resistors, $U^{i}$ is interpreted as the voltage current function, and the same proof shows that a quasi-linear replacement arises.

It is not true that every symmetric semi-definite matrix is a resistor matrix of some network. To state in algebraic terms the restrictions on $R$ to be sure that such a matrix is actually a representation of a network is a topological problem of graphs which has not been solved. The matrix $S$ relating to the voltage drops across the capacitors has exactly the same restrictions and properties as that of $R$.

It is to be noted that the assumption that one or more of the matrices $L, R$, and $S$ could be singular is an idealization. Thus all actual conductors have a nonvanishing resistance, and if this were assumed, it would immediately follow that the matrix $R$ is nonsingular. It has been customary, however, and useful, to assume that some circuits contain, for example, only resistance or only capacitance, and so on. Where this idealization stops is partly a matter of taste, but the weakest assumption which appears to be useful is that the matrix $L+R+S$ is nonsingular.

The nonlinear networks considered previously were assumed to satisfy the equation

$$
L y^{\prime \prime}+V\left(y^{\prime}\right)+S y=e
$$

where $V\left(y^{\prime}\right)$ was taken as a quasi-linear replacement of $R y^{\prime}$. The networks to be discussed here are such that $S=0$. In the linear case the equation satisfied is

$$
L i^{\prime}+R i=e .
$$

In the nonlinear case the equation satisfied is

$$
d V(i) / d t+R i=e
$$

where $V(i)$ is a quasi-linear replacement of $L i$. In order to apply the theory developed in I and III, it is necessary to reduce equation (8) to that of the form of equation (6). Integrating equation (8) gives

$$
V\left(y^{\prime}\right)+R y=p .
$$

Here $p=\int_{0}^{t} e d t+h$ where $h$ is some constant vector of integration. Clearly, (9) is of the form (6) with $L=0$ and $S=R$. 
THEOREM 1. Let $R$ be an arbitrary symmetric definite matrix. Let $e(t)$ be a finite integrable function for $t \geqq 0$. Then if $i_{1}$ and $i_{2}$ are two continuous solutions of equation (8),

$$
\int_{0}^{\infty}\left\|i_{1}-i_{2}\right\|^{2} d t<\infty .
$$

Proof. The hypotheses given are sufficient to insure that relation (9) is valid. Moreover, it may be assumed that $h=0$, because since $R$ is nonsingular, there is a constant vector $k$ such that $R k=h$. Replacing $y$ by $y+k$ achieves this result without changing the value of $y^{\prime}$. Consider now the equation

$$
L y^{\prime}+R y=0 .
$$

In order to apply the main theorem of $I$, it is necessary to know that all solutions of (10) are such that $y^{\prime} \rightarrow 0$ as $t \rightarrow \infty$. To show this, note that in a rotated coordinate system $R$ is a diagonal matrix with positive diagonal elements. Now by making a simple transformation which merely shortens or lengthens the coordinate axes, $R$ can be made to be the identity matrix, $E$. Another rotation of axes converts $L$ to a diagonal matrix. Thus (10) breaks up into $n$ equations of the form $l_{k} y_{k}^{\prime}+y_{k}=0$, where $l_{k} \geqq 0$. Clearly, then, $y_{k}^{\prime} \rightarrow 0$ as $t \rightarrow \infty$. The transformations made in going to this new coordinate system were all nonsingular, so it follows that $y_{k}^{\prime} \rightarrow 0$ in the old coordinate system also. Thus the theorem of $I$ is now directly applicable and completes the proof.

THEOREM 2. Let $R$ be an arbitrary symmetric definite matrix. Let $e(t)$ be a continuous function of period $2 \pi$. Then equation (8) has one and only one solution for which $i$ is a continuous periodic function.

Proof. It is possible to write $e=e_{1}+s$ where the integral of $e_{1}$ over an interval of length $2 \pi$ vanishes and where $s$ is a constant vector. Since $R$ is nonsingular, there is a constant vector $k$ such that $R k=s$. Let $i=i_{1}+k$. Then equation (8) can be written as $d V_{1}\left(i_{1}\right) / d t$ $+R i_{1}=e_{1}$ where $V_{1}$ is the quasi-linear replacement as given by the preceding lemma. Integrating this equation puts it in the form (9), where $p$ is a periodic function with a bounded first derivative. Theorem 5 of III is now directly applicable, and it states that there is a continuous periodic solution $i_{1}$. Theorem 1 , above, implies that there is no other continuous solution of period $2 \pi$ or, for that matter, of any other period. This completes the proof of Theorem 2.

4. Interlinked electric and magnetic networks. The roman num- 
eral III depicts a commonly used transformer core shape. Apparently the analysis given in $\$ 1$ is not applicable for such unsymmetrical transformers. By making use of the familiar concept of a magnetic network, the conclusions of $\$ 3$ will now be shown to remain valid, at the expense, however, of introducing an additional assumption.

A magnetic network is formally analogous to a direct current electric network. First a maximal set of independent circuits is selected in the transformer cores. Then any distribution of flux in the cores can be expressed uniquely as a superposition of cyclic fluxes $f^{j}$ flowing in these circuits. This statement rests on the fact that $\operatorname{div} B=0$. In the linear case the specific magnetic resistance is taken to be $1 / \mu$. In the nonlinear case the specific resistance function is the inverse of the permeability function. The magnetic network equations may then be written in analogy to the electric case. Expressed in vectorial form, they are:

$$
m=M(f) .
$$

Here $m$ may be called the magnetomotive force vector. By definition, the component $m^{i}$ is the line integral of the tangential component of the magnetic field around the $j$ th circuit.

Suppose now that this magnetic network is topologically interlinked with an electric network. It is desired to relate the vectors $m$ and $f$ of "magnetic space" with the vectors $v$ and $i$ of "electric space." Introducing the linkage matrix, $C$, in exact analogy to the matrix $C$ in $\$ 1$, the generalization of relation (1) is:

$$
\text { o }=C f \text {. }
$$

On the other hand, Ampere's line integral law demands that

$$
m=C^{*} i \text {. }
$$

Equations (11), (12), and (13) define an implicit relation between $v$ and $i$. By this method of analysis VerPlanck and Fishman [3] are able to obtain explicit solutions of several magnetic amplifier networks in terms of the basic parameters.

If the magnetic network is constructed of ferromagnetic material, the magnetic resistors may be termed quasi-linear resistors, because the differential resistance will lie between positive limits. The considerations given in $\$ 3$ show that $M$ is a quasi-linear replacement. Since none of the branches have zero resistance, it follows that $M$ has an inverse, $U$. Thus

$$
\vartheta=C U\left(C^{*} i\right)
$$


The differential transformation is $d v=C U^{\prime} C^{*} d i$. But $U$ is a quasilinear replacement, so it is easy to see that the matrix $C U^{\prime} C^{*}$ satisfies conditions (ii) and (iii). Thus (14) defines a quasi-linear replacement in electric space. The theorems in $\$ 3$ are now valid, so it may be said that networks of unsymmetrical transformers and Ohmic resistors are reliable.

The analysis in this section tacitly assumes that it is permissible to treat distributed parameters as mean lumped parameters. A justification of this assumption will be attempted in a later paper. This will involve rather lengthy considerations, because the solution of a nonlinear Dirichlet problem is demanded.

\section{REFERENCES}

1. R. J. Duffin, Nonlinear networks. I, Bull. Amer. Math. Soc. vol. 52 (1946) pp. 833-838.

2. - Nonlinear networks. III, Bull. Amer. Math. Soc. vol. 55 (1949) pp. 119-129.

3. D. W. VerPlanck and M. Fishman, Analysis of interlinked electric and magnetic networks with application to magnetic amplifiers, National Electronics Conference, Chicago, November, 1948.

Carnegie Institute of Technology 